\section{Long exposure of argon plasma coagulation induces more thermal damage accompanied by a higher expression of NF-kB and caspase-3}

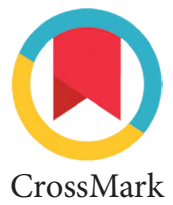

${ }^{1}$ Department of Prosthodontic, Faculty of Dentistry, Hasanuddin University, Makassar, Indonesia ${ }^{2}$ Department of Dentistry, Taipei Medical University Hospital, Taipei, Taiwan

${ }^{3}$ Department of Dentistry, Taipei Medical University-Shuang Ho Hospital, New Taipei City, Taiwan

${ }^{4}$ Department of Dentistry, Health Sciences University of Hokkaido, Hokkaido, Japan

${ }^{5}$ Department of Prosthodontics, Faculty of Dentistry, Universitas Gadjah Mada, Yogyakarta, Indonesia

63D Global Biotech Inc., New Taipei City, Taiwan

${ }^{7}$ Department of Oral and Maxillofacial Surgery, Faculty of Dentistry, Hasanuddin University, Makassar, Indonesia

${ }^{8}$ Department of Dentistry, College of Medicine, China Medical University, Taichung, Taiwan ${ }^{9}$ Biomedical Technology R \& D Center, China Medical University Hospital, Taichung, Taiwan

${ }^{10}$ Department of Dentistry, College of Oral Medicine, Taipei Medical University, Taipei, Taiwan

${ }^{11}$ Dental Department of Wan-Fang Hospital, Taipei Medical University, Taipei, Taiwan

\section{"Corresponding to:}

Chung-Ming Liu, Hsin-Hua Chou,

Department of Dentistry, College of

Medicine, China Medical University,

Taichung, Taiwan. Biomedical

Technology R \& D Center, China Medical University Hospital,

Taichung, Taiwan

Department of Dentistry, College of Oral Medicine, Taipei Medical University, Taipei, Taiwan. Dental Department of Wan-Fang Hospital, Taipei Medical University, Taipei,

Taiwan

liuc@mail.cmu.edu.tw;

hhchou@tmu.edu.tw

\author{
Rahmat A. Waris, ${ }^{1}$ Keng-Liang Ou, ${ }^{1-6}$ Muhammad Ruslin, ${ }^{7}$ Bahruddin Thalib, ${ }^{1}$ \\ Chung-Ming Liu, ${ }^{8,9 *} \mathrm{Hsin}-\mathrm{Hua} \mathrm{Chou}^{10,11^{*}}$
}

\title{
ABSTRACT
}

Objective: Long exposure of argon plasma coagulation (APC) causes thermal damage and apoptosis in tissues. However, whether the APCinduced thermal damage in tissues involves the expression of NF-кB and caspase- 3 remains undetermined. In this study, we compared the effect of APC on liver damage at two different exposure time and tested the hypothesis that thermal injuries induced by APC are accompanied by induction of NF-KB and caspase-3 expression in rat liver.

Material and Methods: Liver injuries were induced in rats by an APC device with pulse mode for 2 or 4 seconds under the same frequency of power (40W). The animals were sacrificed $0,3,7$ and 21 days after injury and the liver tissues were harvested and used for western blotting, histological and immunohistochemical analyses.

Results: Haematoxylin and eosin (H\&E) stained sections of the liver tissues showed that two-second application of APC caused minimum thermal damage and apoptotic areas, less carbonization, and more fibrosis formation in liver than the four-second APC application at all time points examined. All of these APC-induced thermal effects and morphological changes in the two-second APC application group but not the four-second APC application group recovered 21 days after the treatment. Western blot results indicated that APC induced the expression of NF-KB on day 3, and peaked on days and 14. In the twosecond APC application group, the expression of NF- $\mathrm{KB}$ returned to the normal level on day 28. However, the expression of NF- $\mathrm{BB}$ induced by 4 seconds of APC application remained high even 28 days after injury. The expression of caspase- 3 induced by the 2 seconds or 4 seconds of APC application peaked at 7 or 14 days, respectively. Similarly, the APC-induce expression of caspase-3 returned to the normal level in the 2-second APC application group, but it still remained high in the 4-second APC application group even 28 days after injury. These results were further confirmed by The immunofluorescence data also indicated that APC exposure for 4 seconds induced a much higher expression of NF-KB than APC exposure for 2 seconds. The similar pattern was observed in the caspase-3 expression.

Conclusions: Taken together, our results show that 2-second APC exposure causes minimum liver injury accompanied by the expressions of NF-kB and casapase- 3 which return to the normal level 28 days after injury. These findings strongly suggest that the shortest pulse mode ( 2 seconds) application of APC is a safe, convenient, and effective approach for the treatment of particularly thermosensitive tissues.

Keywords: Argon plasma coagulation, Liver, Histology, NF-кB, Caspase-3, Electro surgery

Cite this Article: Waris, R.A., Ou, K., Ruslin, M., Thalib, B., Liu, C., Chou, H. 2018. Long exposure of argon plasma coagulation induces more thermal damage accompanied by a higher expression of NF-KB and caspase-3. Journal of Dentomaxillofacial Science 3(1): 62-69. D0I: $10.15562 / j d m f s . v 3 i 1.734$

\section{INTRODUCTION}

Argon plasma coagulation (APC) is generally believed to be a highly developed and better technique over the contact thermal methods in cauterization and hemostasis during surgery due to its lower risk of perforation and minimum thermal injury. ${ }^{1}$ APC has multiple advantages over the laser and other surgical devices during surgery because it is inert, odorless, easily ionizable, inexpensive and non-contact. Moreover, APC does not generate smoke and carbonization of tissue. ${ }^{2}$ Several studies have reported the use of APC in many applications such as hemostasis of peptic ulcer, tumor ablation, angiodysplasia surgery, and post polypectomy treatment, and gastric cancer treatment. ${ }^{3}$ Treatment with APC alleviates hematochezia induced by chronic radiation proctopathy in prostate cancer patients, and significantly increases hemoglobin levels in these patients. ${ }^{4}$ The APC therapy also attenuates the rectal bleeding and tissue toxicity in chronic radiation proctopathy of patients with cervical cancer. ${ }^{5}$

Many factors affect the thermal damage induced by electrosurgical devices including the power settings, the flow rate of argon gas, the pulse mode and the distance between probe and target tissue. Inappropriate use of electrosurgical devices might cause unintended tissue damage such as denaturation of proteins and collagen and can also cause cell necrosis. A High frequency electrical mode and longer duration pulse mode of APC application can lead to the growth of granulation tissue in the airway in dog model. ${ }^{7}$ A previous study has 
reported that APC-caused longitudinal and circular muscularispropria injury are associated with delivery of energy, and increased depth of tissue damage and surface area of lesions with increased power delivery. ${ }^{8}$ Thermal injury can alter the morphology of liver cells, diminishes protein and DNA concentrations, and modulates hepatic proinflammatory cytokines such as: NF- $\kappa \beta,{ }^{9}$ and caspase- $3 .{ }^{10}$ It was reported that caspase- 3 regulates DNA fragmentation and morphological change for apoptosis. ${ }^{11}$ Thermal injury induces apoptosis via inhibition of NF- $\kappa \beta$ and caspase- 3 expression in liver. ${ }^{12}$ These results suggest that thermal injury associated with expression of NF- $\kappa \beta$ and caspase- 3 may lead to apoptosis.

Several clinical and experimental studies have authenticated that APC-caused thermal damage, and perforation is mostly dependent on setting of power and pulse duration. APC induces severe gastric mucosal damage associated with pulse duration (pulse mode) and electrical power setting (precise mode), and the maximum esophageal mucosal injuries are associated with APC pulse duration. ${ }^{13}$ Application of APC in seconds has shown an effective treatment for premalignant and malignant lesion of upper gastrointestinal tract. ${ }^{14} \mathrm{It}$ was reported that APC with power setting $(155 \mathrm{~W})$ and pulse duration (10 seconds) causes minimum lesions ${ }^{15}$ and reduces severity of tissue coagulation. A clinical report indicated that application of intraductal APC with one second for ablation of biliary neoplasia in patient. ${ }^{16} \mathrm{~A}$ recent study has reported that APC with power setting $40 \mathrm{~W}$ and two second pulse mode could not cause any adverse effect for treatment of submucosal telangiectasia. ${ }^{17}$ Several reports have confirmed the efficacy of APC based on power setting and pulse duration which may contribute to the reduced high risk of thermal damage. Till date, studies have not been done to compare the different pulse modes of APC-caused thermal damage, apoptosis in liver tissues via the possible mechanisms with NF- $\kappa \mathrm{B}$ and caspase-3 expression. Hence, for the first time that we have evaluated the efficacy of APC with different pulse mode related complications and comparison was performed to evaluate the thermal damage outcome in the rat liver tissue.

\section{MATERIAL AND METHODS}

\section{Animals}

The animal experiments were reviewed and approved by the Institutional Animal Care and Use Committee for Taipei Medical University (LAC-99-0037). Totally 16 Sprague-Dawley (SD) rats (weighted 200-300 g, BioLASCO, Taiwan) were used and maintained in accordance with the guidelines for the care and use of laboratory animals. The animals were fed ad libitum and housed in a temperature, humidity and light-controlled environment. Rats were marked to permit individual identification, and kept in their cages for 14 days prior to the beginning of experiments to allow for acclimatization to the laboratory conditions.

\section{Surgical procedure}

General anesthesia was induced with inhaled isoflurane of the animals. The operative site was cleansed and draped in a sterile fashion. Conventional stainless steel (SS) needle electrodes was used with an electrosurgical unit. The liver was exposed through midline laparotomy with a retractor to avoid injury to the liver. The APC was treated to the liver lobe to create an injury using a fixed power setting (40 watts) with different pulse duration (two seconds and four seconds). Pulse mode 2 (2 pulse per 000 milliseconds or $0.0 \mathrm{~Hz}$ ) and pulse mode 4 ( 2 pulse per 000 milliseconds or $0.0 \mathrm{~Hz}$ ) were used with similar power setting. All thermal injuries were created with the probe at a distance of 2 to $3 \mathrm{~mm}$ from the target tissue.

\section{Injury area and histological observation}

Histopathological studies were performed to provide structural evidence of the surgery mediated tissue injury. Animals were sacrificed on days $0,3,7$ and 21 post-operation, respectively. Tissues were then cut into pieces of desired size and fixed in Bouin's fluid fixative immediately after autopsy. Fixation was carried out at room temperature for $24 \mathrm{hr}$, after which the tissues were transferred to $70 \%$ alcohol. Several changes of $70 \%$ alcohol were given until the yellow color disappeared from the tissues. The tissues were then dehydrated by passing through ascending grades of alcohol (30\%, $50 \%, 70 \%, 90 \%$ and $100 \%$ ), cleared in methyl salicylate and infiltrated with wax at $57^{\circ} \mathrm{C}$. The tissues thus cleared were embedded in the paraffin. Conventional techniques of paraffin -wax secondstioning and haematoxylin-eosin staining were used for histological studies. ${ }^{18}$ Paraffin sections of 6 to $8 \mu \mathrm{m}$ thickness were cut using a rotary microtome (Leica, Germany). The sections, thus obtained, were stained in Harris' haematoxylin and eosin and then washed in 90\% alcohol for a few seconds. The stained sections were dehydrated in $100 \%$ alcohol, cleared in xylene and mounted in DPX mountant. The stained slides were observed in a Carl Zeiss (Germany) Axio 2 Plus Research Microscope. Images were captured through a CCD camera (BX51, Olympus, Japan) in a computer and processed using Carl Zeiss Axiovision software. The total injury area caused by the SS-needle was measured using image analysis software (SPOT 
basic software, SPOT imaging solutions, MI, USA). In addition, different histological features were observed in the injury site. Therefore, the injured area was further divided into different classifications to demonstrate the injury caused by thermal spread, and the area of every portion was measured.

\section{Western blot analysis}

The retrieved liver tissues were homogenized and lysed in an ice-cold buffer and centrifuged at $13,000 \mathrm{rpm}$ for $15 \mathrm{~min}$ at $4^{\circ} \mathrm{C}$. Protein concentrations of the lysates were determined using a Bio-Rad protein assay (Bio-Rad Laboratories, Hercules, CA, USA). The lysates $(20 \mu \mathrm{g} / \mathrm{lane})$ were resolved by sodium dodecyl sulfate (SDS)-polyacrylamide gel electrophoresis (PAGE) in 10\% gels and electroblotted onto a nitrocellulose membrane (HybondTM-C Extra, Amersham Biosciences Corp., Hong Kong, China). The membrane was blocked for $30 \mathrm{~min}$ at room temperature in $3 \%$ nonfat milk in Trisbuffered saline with $0.05 \%$ Tween 20 , and then incubated with the indicated primary antibodies against NF- $\mathrm{B}$ (1:500, MAB3026, Chemicon, Millipore, Temecula, CA) at $4^{\circ} \mathrm{C}$ for overnight and then with horseradish peroxidase-conjugated secondary antibodies at room temperature for $2 \mathrm{~h}$. After washing, the membrane was developed using chemiluminescence substrates (WBLUC0100, Millipore, Temecula, CA). Densitometric analysis of the gels was performed using Gei-Pro analyzer software, and the results were expressed as relative to the density of the $\beta$-actin (1:500, MAB1501, Chemicon, Millipore, Temecula, CA).

\section{Immunofluorescence staining}

For immunofluorescence staining, liver tissues were embedded in Cryomatrix (Thermo Shandon, Pittsburgh, PA, USA) and stored at $-80^{\circ} \mathrm{C}$. Cryostat secondstions (5-7 $\mathrm{mm}$ thickness) were fixed in a mixture of methanol and acetone $(1: 1)$ at $-20^{\circ} \mathrm{C}$ for $5 \mathrm{~min}$ and incubated in 1\% Triton X-100 for another $5 \mathrm{~min}$. Blocking of nonspecific activity was accomplished by incubating the sections with $10 \%$ horse serum in a buffer containing $0.02 \%$ Tween 20 in phosphate-buffered saline for $30 \mathrm{~min}$. Sections were then incubated with primary antiCaspase- 3 for overnight at $4^{\circ} \mathrm{C}$. The localization of antigen was indicated by a Cy5 (red fluorescence, 111-176-003, Jackson ImmunoResearch, West Grove, PA). Finally, the samples were stained with 4', 6-diamidino-2-phenyl-indole (DAPI, sc-3598, Santa Cruz Biotechnology, CA) for $7 \mathrm{~min}$, and examined using a fluorescent microscope. Normal liver tissues obtained from healthy rats also underwent identical procedure. The similar protocol was followed to process negative controls, except that the primary antibodies were replaced as IgG. In situ cell death detection kit (terminal deoxynucleotidyl transferased UTP nick end labeling, TUNEL, 11684795910, Roche, Mannheim, Germany) was used to detect the apoptotic cells, and the apoptotic cells were indicated by a FITC fluorochrome (green fluorescence).

\section{Statistical Analysis}

Data were expressed as mean \pm standard error of the mean (SEM). Statistical analysis was carried out by using analysis of variance (ANOVA). All statistical analyses were performed using SPSS version 12.0 (SPSS, Inc, Chicago, IL). The difference was considered significant when the p-value was less than 0.05

\section{RESULTS}

\section{Effects of APC pulses on thermal injury in liver tissue}

Figure 1 and 2 show the histolopathological alterations in liver tissues of two and four second application of APC in 0, 3, 7 and 21 days. The tissue fluid and accumulation of immune cells were increased in two second application of APC at day 0 than in four second application (shown by red arrows). The injured area was also decreased in pulse mode 2 when compared with pulse mode 4 in day 0 . The bleeding areas were also observed in 4 seconds pulse mode application. Moreover, in a day of 3 and 7 , an increased level of carbonized tissue, macrophage phagocytosis and apoptotic cell dispersion were obviously appeared in 4 seconds pulse mode application figure 1 black arrows. Moreover, application of APC on day 21 in both 2 and 4 seconds of pulsed were created fibrosis formation, suggested that the increased duration of the pulse may cause thermal tissue injury via fibrosis formation figure 2. These results clearly highlight that APC causes less thermal injury in liver tissue during the application of shortest pulse duration.

\section{Effects of APC pulse mode on apoptosis and fibrosis formation in liver tissue}

Morphological changes were observed to determine whether APC induced thermal injury is associated with programmed cell death or apoptosis and fibrosis formation in the liver tissue. As expected, less carbonized tissue and apoptosis cells (shown by black arrows) were observed in two seconds of APC application at day 7 figure 3 , whereas high apoptosis cells (indicated with red arrows) were observed in four seconds of APC application figure 4C and 4D. Apoptosis was observed in both groups, but the minimum apoptosis were observed in pulse mode 


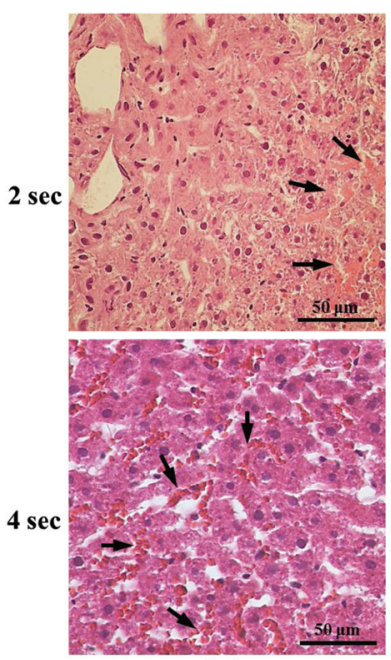

0 day

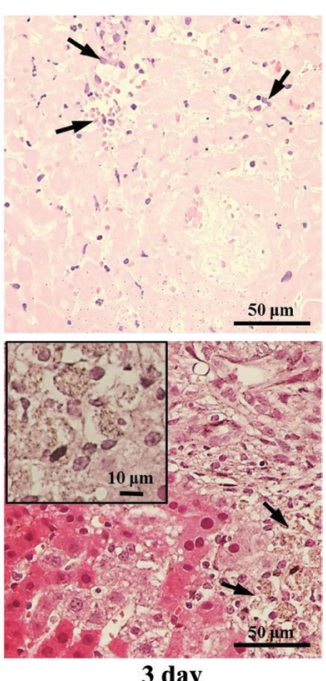

3 day
Figure 1 Photomicrographs of rat liver tissue lesions created by argon plasma coagulation (APC) at the power of $40 \mathrm{~W}$ for 2 and 4 seconds. The tissue fluid and immune cells are highly accumulated in two second groups than four seconds and there were shown with red arrows The carbonized tissue and macrophage phagocytosis in four second group were displayed with black arrows. (H\&E, orig. mag. $\times 40)$

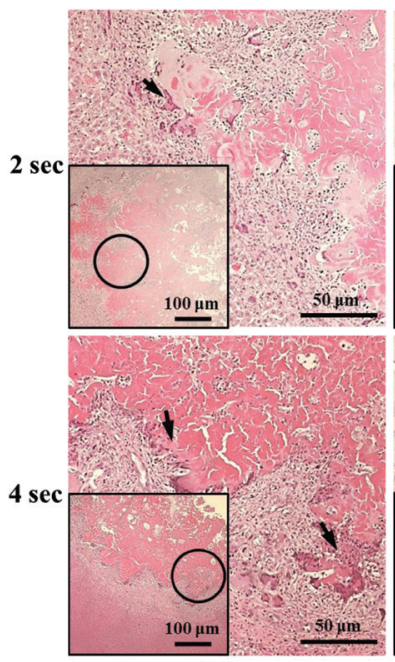

7 day

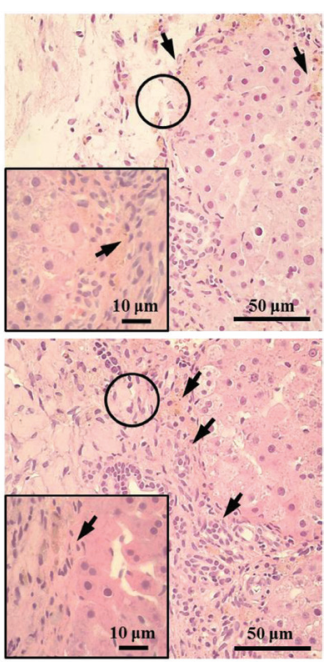

21 day
Figure 2 Photomicrograph of rat liver tissue lesion created by APC at power of $40 \mathrm{~W}$ for 2 and 4 seconds. Here, the less carbonized tissue in two second pulsed groups was shown with black arrows. The red arrows indicate the high apoptosis cells occurred in four second pulsed groups as compared with two second group. (H\&E, orig. mag. $\times 40)$

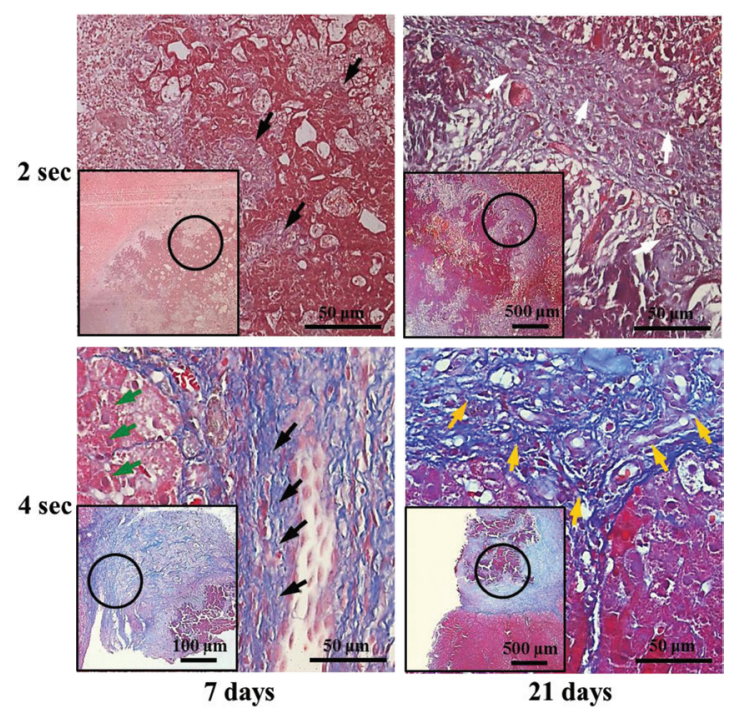

Figure 3 Photomicrograph of rat liver tissue lesion created by APC at power of $40 \mathrm{~W}$ for 2 and 4 seconds. Green arrows indicate that healing process and moderate levels of fibrosis formation in two second groups, whereas the black arrow shows high levels of fibrosis in four second group. (H\&E, orig. mag. $\times 40)$

2 when compared with pulse mode 4 of APC. These results suggest that apoptosis area reduces when APC was used with shortest pulse duration.

Further, we evaluated the fibrosis formation in the thermal injury area after APC-treated with different pulse mode. Increased fibrosis formation was observed in the thermal injury area in four second application of APC at day 7 figure 3 , and the moderate level of fibrosis formation was occurring in the thermal injury area with two second application of APC at day 7 figure 3. These results suggest that APC caused cell death, bleeding, and fibrosis formation is associated with pulse duration of APC application.

\section{APC pulse mediated thermal damage in liver tissue is associated with NF- KB expression}

This study was performed to evaluate whether pulse induced thermal damage in liver tissue is associated with NF- $\kappa B$ expression. The results revealed that 2 seconds pulsed APC decreases the expression of NF- $\kappa B$ in liver tissue than that of 4 seconds pulse mode. The NF- $\kappa \mathrm{B}$ protein expression was moderately increased at days 3 and 7 , and it was peaked at day 14. However, NF- $\kappa$ B expression was gradually reduced after the 21 days in both groups figure 4 . These results were confirmed by densitometric analysis. Normally, NF- $\kappa B$ can be activated by an appropriate stimulus and returns 

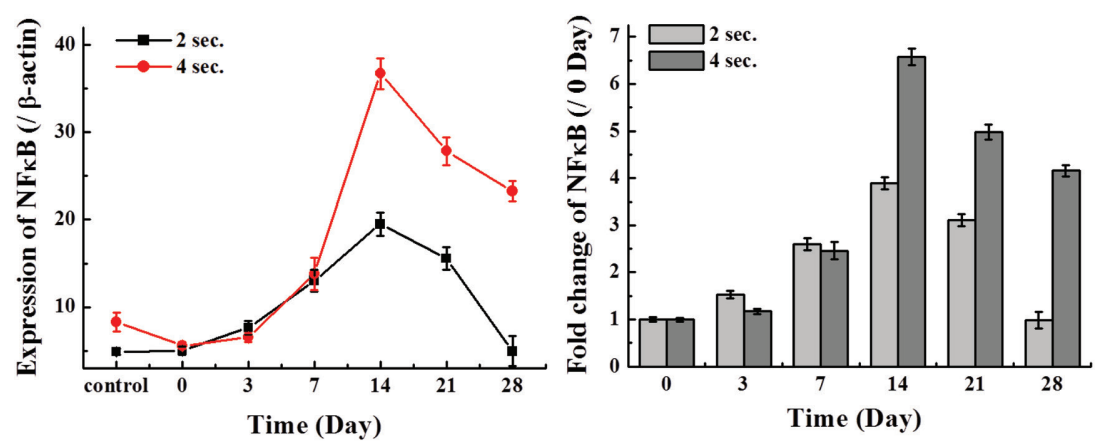

Figure 4 The relative expressions of NF-kB in APC (power of $40 \mathrm{~W}$ for 2 and 4 seconds, at days 3-28) treated rat liver tissue. It shows the relative densitometric analysis of Western blotting

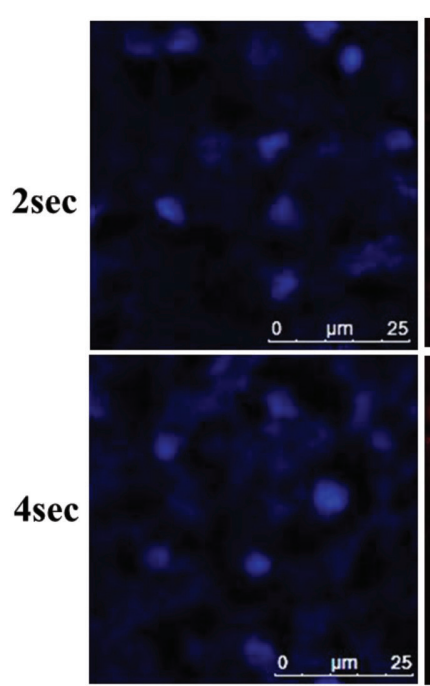

DAPI
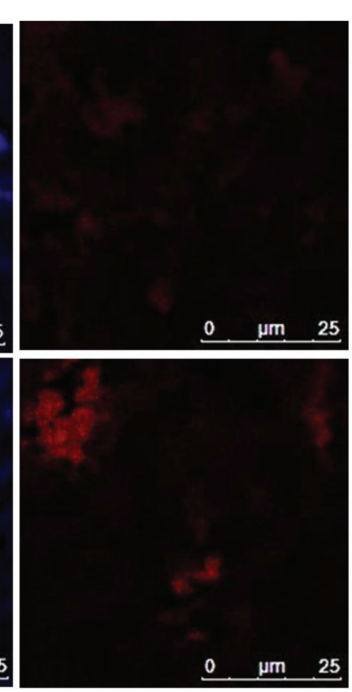

Cy5-NFкB
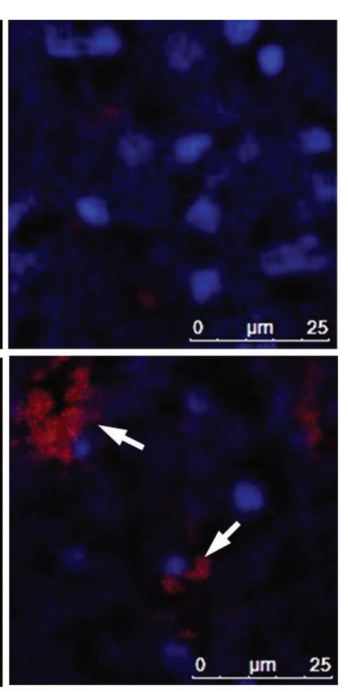

Merge
Figure 5 The immunofluorescence staining data of NF-kB in APC treated rat liver tissue. White arrows indicate that NF-kB is highly dispersed in 4 seconds APC treated groups
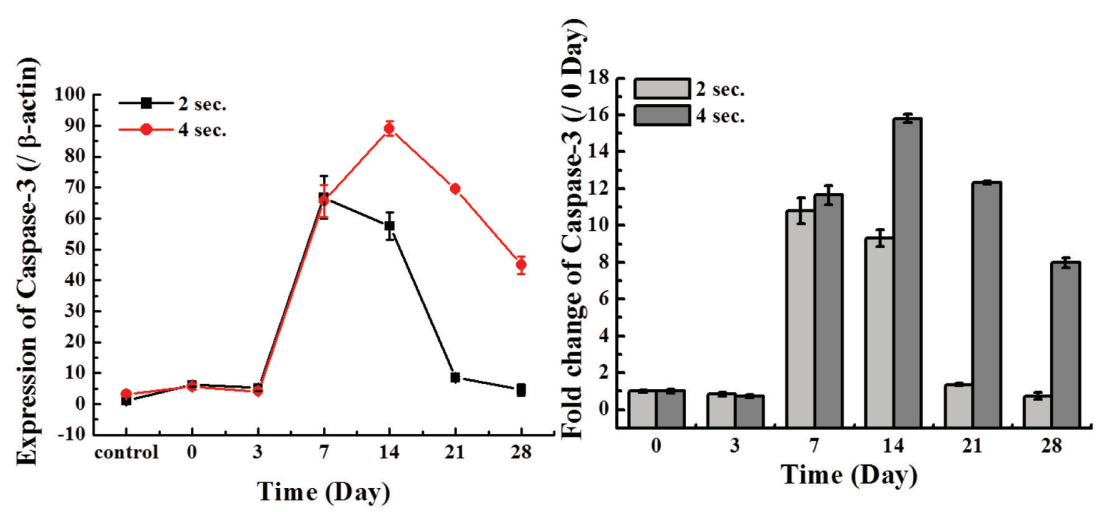

Figure 6 The relative expressions of caspase- 3 in APC (power of $40 \mathrm{~W}$ for 2 and 4 seconds, at days 3-28) treated rat liver tissue. It shows the relative densitometric analysis of Western blotting

to the inactive status after the transcription in the target genes have been completed. This might be the reason that the expression of NF- $\kappa B$ is reduced after 14 days of APC application. This result was also further authenticated by immunostaining and it was demonstrated that the distribution of NF- $\mathrm{kB}$ and inflammatory response were significantly increased in 4 seconds pulsed APC, whereas it was more prominently reduced in 2 second pulsed APC figure 5.

\section{Effects of APC pulse mediated thermal damage on caspase- 3 expression}

Since, the caspase- 3 family appears to play a dominant role in the downstream signaling of apoptotic death ${ }^{19}$ and in the apoptotic liver injury to TNF- $\alpha,{ }^{20}$ we examined the degree of apoptosis in APC caused thermal injury in liver tissues. In this study caspase-3 expression was highly expressed at day 7 and 14 of 4 seconds pulsed APC than in 2 seconds. Further, the increase in caspase- 3 activity was gradually decreased after 7 days and it was highly disappeared at 28 days after APC application in both 2 and 4 seconds pulsed APC figure 6. This result was confirmed by densitometric analysis and these results suggest that the different setting pulse mode of APC induced thermal injury is dependent in caspase-3 expression in liver tissue.

\section{DISCUSSION}

This is the first study to characterize whether the thermal injury in the liver in response to APC under different conditions of pulse duration and at the same power output associate with NFK-B and caspase- 3 expressions. The results demonstrate that at the same power settings (40W) and 4 seconds pulsed duration used in the liver tissues during monopolar electrosuregery, APC is capable of causing significant damage to the liver through inducing over expression of NFK-B and caspase-3. APC is a relatively economical, simple, safe, and adaptable technique. Numerous endoscopic applications have been advocated, as they are generally subdivided into vascular coagulation and tissue ablation. At low power settings, APC has been reported to be effective therapy for a wide variety of vascular lesions including angiodysplasia, ${ }^{21,22}$ watermelon stomach, ${ }^{23,24}$ and radiation proctopathy. ${ }^{25,26}$ Even though there are few data from randomized comparative trials of APC versus other modalities, such as heat probe, monopolar electrosurgical, and laser coagulation, it is believed that the depth of injury with APC is less than that of these other techniques, and that APC is therefore less likely to result in postcoagulation syndrome. Several studies of the depth of injury in the colon associated with monopolar electrosurgical and laser coagulation have demonstrated significant damage to the muscularis propria and serosa. ${ }^{27}$ One small study demonstrated equivalence between APC and captive coagulation with the heat probe 
in the management of peptic ulcer hemorrhage. ${ }^{28}$ Another report indicated that APC induces severe gastric mucosal damage when pulse duration and electrical power setting are increased. ${ }^{13}$ High power setting of APC caused high scores of circular muscle and longitudinal muscle injuries, and lesions, ${ }^{6}$ and the depth of injuries and the surface area of lesions depends on the power setting, power delivery and pulse mode. ${ }^{8}$ These results are highly harmony with the present study, as it demonstrated that APC with 4 seconds pulse mode increases thermal injury area than shorter pulse mode application. The results of this study have suggested that the pulse duration of APC plays significant role in tissue damage during surgery, and application of APC with high pulse mode causes high width of the thermal injury area of liver tissue. Similarly, the more recent study of Watson et al., ${ }^{13}$ which used resection specimens of esophagus and stomach, found that APC at a power setting of $99 \mathrm{~W}$ for 3 seconds caused injury to the muscularis propria in only $5 \%$ of samples. The results of the present study have also established that high tissue fluid or edema, and immune cells were observed around the thermal injured area in two seconds of APC application than that of four seconds duration. These results are in agreement with other studies, where they reported that increase of edema in pancreas and fluid accumulation around the pancreatic head after treatment with APC for ablation of duodenal adenomatous polyps. ${ }^{29}$ The same study reported that application of APC with high precise mode caused the deep lesions, granulation tissue formation and inflammation in rat peritoneal trauma sites. ${ }^{29}$ With these results, we suggest that the severity of thermal damage, tissue carbonization and apoptosis corresponds with pulse duration of APC application and the short pulse mode of APC causes less thermal damage area, apoptosis and high fibrosis formation in the liver tissue.

NF- $\kappa \mathrm{B}$ activation plays a role in the regulation of more than 400 gene product expressions associated with inflammation, cell survival, proliferation, invasion and angiogenesis. ${ }^{20,31}$ NF-қB normally binds to ІқВ $\alpha$, which impedes NF-қB nuclear translocation from the cytoplasm to the nucleus. NF-kB is reported to be a vital link between hepatic injury, fibrosis and even hepatocellular carcinoma, ${ }^{32}$ it enters the nucleus, with the degradation of $\mathrm{IkB}$ and activates the expression of specific genes that induce the apoptosis cascade. ${ }^{33}$ Once cells are exposed to inflammatory stimuli, including LPS and TNF- $\alpha$, Іқ $\mathrm{B} \alpha$ is phosphorylated, leading to ІқB $\mathrm{B}$ degradation and nuclear translocation of NF-қB. In our previous study, it was noticed that liver tissues that had been operated by SS-needles secreted higher levels of NF-kB expression and its nuclear translocation than that of $\mathrm{CrN}$ operated tissues. ${ }^{34}$ In this study, we thus evaluated whether an APC application with two different pulse durations have any effect on protein expression and nuclear dispersion of NF- $\mathrm{KB}$ during 2 and 4 seconds pulsed APC and the results we found for the first time that short duration pulse APC mode expresses lower expression of NF-kB and its distribution in hepatic cells. Normally, NF$\kappa \mathrm{B}$ can be activated by an appropriate stimulus and returns to the inactive status after the transcription in the target genes have been completed. This might be the reason that the expression of NF- $\kappa B$ was peaked on day 14 and the gradually decreased until 28 days. Yates and Rayner, ${ }^{35}$ studied the effects of fatty acid supplementation on activation of NF-kB and AP-1 after a skin injury, since these transcription factors are highly associated with wound healing. They have also reported a novel finding which indicates that wounding of normal human keratinocytes in culture causes NF-kB translocation. The relevance of NF-kB activation to wound healing is suggested by the results that HeNe laser treatment, a process shown to accelerate healing in a model of wounding, ${ }^{36}$ promoted the translocation of NF-kB. One another study reported that NF- $\mathrm{KB}$ expression dramatically increased in the thermal injury area. ${ }^{9}$

Apoptosis is involved in the regulation of collagen synthesis and degradation within the wounds through the regulation of fibroblast numbers and collagenase activity. ${ }^{37}$ Recent work in another mouse model suggests that hypertrophic scars demonstrate decreased rates of apoptosis, with decreased expression of cleaved caspase 3. Over expression of various caspases family member induces apoptosis in cultured mammalian cells. ${ }^{38}$ Among the cysteine proteases, caspase- 3 is believed to be one of the most commonly involved in the execution of apoptosis in various cell types. ${ }^{39}$ It was reported that caspase- 3 induces programmed cell death via its intrinsic and extrinsic pathways and degradation of protein and DNA in cells, ${ }^{40}$ and it has also reported that burn injury induces apoptotic proteins such as: bax, bcl-2 and caspase- 3 in skeletal muscles. ${ }^{10}$ It was reported that treatment of non-thermal plasma induce caspase-3 cleavages in melanoma cancer cells. ${ }^{41}$ These evidences clearly implicated that thermal injury induces caspase- 3 that employ as direct evidence whether cell death occurred by apoptosis. In this study, low level of caspase- 3 was expressed in 2 seconds of APC application than in 4 seconds, and it gradually reduced to basal level at 21 and 28 days.

\section{CONCLUSION}

This is the first study to determine the characteristics prevailing intensity of thermal injury to the 
liver tissues with 2 and 4 seconds pulsed APC. At a constant power setting $(40 \mathrm{~W})$ with 4 seconds pulse caused a significant damage to liver tissues, such as thermal injury, less carbonized tissue and apoptosis, increased fibrosis formation and lower the accumulation of immune cells. Thermal injury caused by the increased duration of APC pulse mode could partially involved in the expression of NF-kB and caspase-3. These data suggest that APC should be used with care, principally at low pulse mode settings in the liver. This study may also provide a better understanding of the scientific validation of the therapeutic value of the low pulse mode APC application in therm al sensitive tissues, particularly hepatic thermal injury.

\section{CONFLICT OF INTEREST}

The authors report no conflict of interest in this work.

\section{REFERENCES}

1. Canard JM, Vedrenne B, Bors G, et al. Treatment of radiation proctitis by argon plasma coagulation: Long term results. Gastroenterologie Clinique et Biologique 2003;27: 455-459.

2. Miyazawa TH, Nawashiro H, Shima K, et al. Early experiences of haemostasis on brain tumour surgery with argon plasma coagulation. Acta Neurochir (Wi;en) 2000;142: 1247-1251.

3. Vargo JJ. Clinical applications of the argon plasma coagulator. Gastrointest Endosc 2004; 51: 81-88.

4. Alfadhli AA, Alazmi WM, Ponich T, et al. Efficacy of argon plasma coagulation compared with topical formalin application for chronic radiation proctopathy. Can J Gastroenterol 2008;22: 129-132.

5. Villegas JC, Cossio SS, Téllez LC, et al. Argon plasma coagulation and hyperbaric oxygen therapy in chronic radiation proctopathy, effectiveness and impact on tissue toxicity. Revista Espanola de Enfermedades Digestivas 2011; 576-581.

6. Norton ID, Wang L, Levine SA, et al. In vivo characterization of colonic thermal injury caused by argon plasma coagulation. Gastrointest Endosc 2002;55: 631-636.

7. Zhang J, Wang T, Ting W, et al. Effect of three interventional bronchoscopic methods on tracheal stenosis and the formation of granulation tissues in dogs. Chinese Medical Journal 2010;123: 621-627.

8. Goulet CJ, DiSario JA, Emerson L, et al. In vivo evaluation of argon plasma coagulation in a porcine model. Gastrointest Endosc 2007;65: 457-462.

9. Jeschke MG, Aililow JF, Randi VMS, et al. Cell proliferation, apoptosis, NF-kB expression, enzyme, protein, and weight changes in livers of burned rats. Am J Physiol Gastrointest Liver Physiol 2001;280: G1314-G1320.

10. Duan $\mathrm{H}$, Chai j, Sheng Z, et al. Effect of burn injury on apoptosis and expression of apoptosis-related genes/ proteins in skeletal muscles of rats. Apoptosis 2009;14: 52-65.

11. Slee EA, Adrain C, Martin SJ. Executioner caspase-3, -6 , and -7 perform distinct, non-redundant roles during the demolitionphase of apoptosis. J Biol Chem 2001;276: 7320-7326.

12. Ipaktchi K, Mattar A, Niederbichler AD, et al. Topical p38 MAPK inhibition reduces dermal inflammation and epithelial apoptosis in burn wounds. Shock 2006;26: 201-209.
13. Watson JP, Bennett MK, Griffin SM, et al. The tissue effect of argon plasma coagulation on esophageal and gastric mucosa. Gastrointest Endosc 2000;52: 342-345.

14. Manner H, May A, Faerber M, et al. Safety and efficacy of a new high power argon plasma coagulation system (hp-APC) in lesions of the upper gastrointestinal tract. Digestive \& Liver Disease 2006;38: 471-478.

15. Johanns W, Luis W, Janssen J, et al. Argon plasma coagulation (APC) in gastroenterology: experimental and clinical experiences. Eur J Gastroenterol Hepatol 1997;9: 581-587.

16. Jazrawi SF, Nguyen D, Barnett C, et al. Novel application of intraductal argon plasma coagulation in biliary papillomatosis (with video). Gastrointest Endos 2009;12: 372-374

17. Sato Y, Takayama T, Sagawa T, et al. Argon plasma coagulation treatment of hemorrhagic radiation roctopathy: the optimal settings for application and long-term outcome. Gastrointest Endosc 2011;73: 543-549.

18. Drury RAD, Wallington EA. Carleton's Histological Technique: 1981.

19. Enari M, Talanian RV, Wong WW, et al. Sequential activation of ICE-like and CPP32-like proteases during Fasmediated apoptosis. Nature 1996;380: 723-726.

20. Kunstle G, Leist M, Uhlig S, et al. ICE-protease inhibitors block murine liver injury and apoptosis caused by CD95 or by TNF-alpha. Immunol Lett 1997;55: 5-10.

21. Norton ID, Geller A,Viggiano TR, et al. The argon plasma coagulator: efficacy and safety profile [abstract]. Gastrointest Endosc 1999;49: AB130.

22. Bergler W, Riedel F, Baker-Schreyer A, et al. Argon plasma coagulation for the treatment of hereditary hemorrhagic telangiectasia. Laryngoscope 1999;109: 15-20.

23. Focke G, Seidl C, Grouls V. Treatment of watermelon stomach (GAVE syndrome) with endoscopic argon plasma coagulation (APC). A new therapy approach (in German with English abstract). Leber, Magen, Dann 1996;26: 254,257-259.

24. Saurin J-C, Cohelo J, Lepretre J, et al. Argon plasma coagulator efficiently controls bleeding in patients from watermelon stomach and radiation proctopathy [abstract]. Gastrointest Endosc 1999;49: AB169.

25. Silva RA, Correia AJ, Dias LM, et al. Argon plasma coagulation therapy for hemorrhagic radiation proctosigmoiditis. Gastrointest Endosc 1999;50: 221-224.

26. Fantin AC, Binek J, Suter WR, et al. Argon beam coagulation for treatment of symptomatic radiation-induced proctitis. Gastrointest Endosc 1999;49: 515-518.

27. Savides TJ, See JA, Jensen DM, et al. Randomized controlled study of injury in the canine right colon from simultaneous biopsy and coagulation with different hot biopsy forceps. Gastrointest Endosc 1995;42: 573-578.

28. Cipolletta L, Bianco MA, Rotondano G, et al. Prospective comparison of argon plasma coagulator and heater probe in the endoscopic treatment of major peptic ulcer bleeding. Gastrointest Endosc 1998;48: 191-195.

29. Kraemer A, Baskol M, Gursoy S, et al. Epinephrine plus argon plasma or heater probe coagulation in ulcer bleeding. World J Gastroenterol 2011;17: 4109-4112 .

30. Dolcet D, Llobet J, Pallares X, et al. NF-kB in development and progression of human cancer", Virchows Archiv : an International Journal of Pathology 2005;446: 475-482.

31. Gilmore TD. Introduction to NF-kappaB: players, pathways, perspectives. Oncogene 2006;25: 6680-6684.

32. Luedde T, Schwabe RF. NF-kappaB in the liver-linking injury, fibrosis and hepatocellular carcinoma. Nature reviews Gastroenterology \& Hepatology 2011;8: 108-118.

33. Schmid JA, Birbach A. IkappaB kinase beta (IKKbeta/ IKK2/IKBKB) - a key molecule in signaling to the transcription factor NF-kappaB. Cytokine \& Growth Factor Reviews 2008;2: 157-165.

34. Chiang HJ, Chou HH, Chiou SY. In vivo study on biomedical devices with nanostructured composite films in minimal invasive surgery. Third International Workshop on Nanotechnology and Application (IWNA 2011), Vung Tau, 2011. 
35. Yates S, Rayner TE. Transcription factor activation in response to cutaneous injury: role of AP-1 in reepithelialization. Wound repair and Regeneration 2002;10: 5-15.

36. Haas AF, Isseroff RR, Wheeland RG, et al. Low-energy helium-neon laser irradiation increases the motility of cultured human keratinocytes. The Journal of Investigative Dermatology 1999; 94: 822-826.

37. Bian J, Sun Y. Transcriptional activation by $p 53$ of the human type IV collagenase (gelatinase A or matrix metalloproteinase 2) promoter. Molecular and Cellular Biology 1997; 17: 6330-6338

38. Wang S, Miura M, Jung Y. et al., Identification and characterization of Ich-3, a member of the interleukin-1beta converting enzyme (ICE)/Ced-3 family and an upstream regulator of ICE. The Journal of Biological Chemistry 1996;271: 20580-20587.
39. Alnemri ES, Livingston DJ, Nicholson DW et al., Human ICE/CED-3 protease nomenclature Cell 1996;87: 171.

40. Elmore S. Apoptosis: a review of programmed cell death. Toxicol Pathol 2007;35: 495-516.

41. Sensenig R, Kalghatg A, Ccerchar E, et al. Non-thermal Plasma Induces Apoptosis in Melanoma Cells via Production of Intracellular Reactive Oxygen Species. Annals of Biomedical Engineering 2011;39: 674-687.

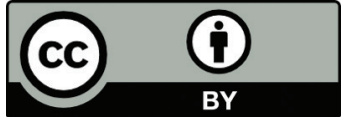

This work is licensed under a Creative Commons Attribution 